\title{
Experimental Evaluation of MIMO Coded Modulation Systems: are Space-Time Block Codes Really Necessary?
}

\author{
Francisco J. Vázquez Araújo ${ }^{1}$, José A. García-Naya ${ }^{1}$,Miguel \\ González-López ${ }^{1}$, Luis Castedo ${ }^{1}$ and Javier Garcia-Frias ${ }^{2}$ \\ ${ }^{1}$ University of A Coruña \\ ${ }^{2}$ University of Delaware \\ ${ }^{1}$ Spain \\ ${ }^{2}$ USA
}

\section{Introduction}

The use of multiple transmit and/or receive antennas, referred to as Multiple-Input Multiple-Output (MIMO) systems, is one of the most promising transmission techniques for achieving the high data rates demanded by the future wireless communication systems. This assertion relies on the theoretical and experimental evidence that the capacity of a MIMO system is considerably higher than that of a conventional single antenna system (Telatar, 1995).

Extracting the maximum capacity and diversity from the MIMO channel requires specific coding techniques that spread channel symbols over both spatial and temporal dimensions of the MIMO channel. The Alamouti code (Alamouti, 1998) is one of the most widely used Space-Time Block Codes (STBC) because of its low encoding and decoding complexity, and its ability to provide the maximum transmit diversity. For these reasons, it has been adopted by the IEEE 802.16-2009 standard (WiMAX) for wireless local and metropolitan area networks (IEEE Standard for Local and Metropolitan Area Networks. Part 16: Air Interface for Fixed and Mobile Broadband Wireless Access Systems, 2009), as well as in the recently approved IEEE 802.11n (WiFi) next-generation wireless standard for Local Area Networks (IEEE Standard for Wireless LAN Medium Access Control (MAC) and PHYsical Layer (PHY) Specifications: Amendment: Medium Access Control (MAC) Enhancements for Higher Throughput, 2009).

The utilization of the Alamouti code is limited to the case of two transmit antennas (i.e., $n_{T}=2$ ) but it does not impose any constraint into the number of receive antennas (i.e., $\left.n_{R}\right)$. However, information-theoretic analysis show that the signal structure imposed by the Alamouti code reduces the capacity of the MIMO channel when there is more than one receiving antenna (Sandhu \& Paulraj, 2000). In the particular case of $2 \times 2$ MIMO systems, this limitation is overcome with the utilization of the Golden code (Belfiore et al., 2005). The Golden code is another example of STBC and constitutes an appealing alternative to the Alamouti code since it does not suffer from capacity loss and exhibits a reasonable complexity cost. 
In spite of their attractive properties, STBCs need an outer channel code to approach the theoretical capacity limit of a MIMO channel since STBCs provide little (or no) coding gain. Remarkable coding gains can be obtained if a capacity-approaching binary encoder, such as Turbo (Berrou et al., 1993) or Low-Density Parity Check (LDPC) (Gallager, 1963; MacKay, 1999), is employed. In this chapter, we focus on a particular subclass of LDPC codes known as Serially-Concatenated Low-Density Generator Matrix (SCLDGM) codes (Garcia-Frias \& Zhong, 2003), whose performance is similar to that of general LDPC codes but with very low encoding complexity. Alternatively, Irregular Repeat-Accumulate (IRA) codes (Jin et al., 2000) can also be used (ten Brink \& Kramer, 2003; Yue \& Wang, 2005), but SCLDGM codes are preferable because their regular versions already approach the capacity limit.

Without using the aforementioned STBCs, the capacity of a MIMO channel can be approached for an arbitrary number of transmitting and receiving antennas by spatially multiplexing the output of a Bit-Interleaved Coded Modulation (BICM) scheme (Tonello, 2000; Zehavi, 1992), constructed with a properly designed capacity-approaching code, i.e. with a capacity-approaching code specifically designed to match the EXtrinsic Information Transfer (EXIT) (ten Brink, 2001) characteristic of the channel. The main difficulty when implementing BICM with spatial multiplexing is the complexity of the detection stage. In general, complexity of optimum detection (Log-Likelihood Ratio (LLR) computation) in BICM with spatial multiplexing is considerably higher than that in systems using STBCs, and it is only affordable for a moderate number of antennas and small constellation sizes.

When detection complexity grows excessively and the number of receiving antennas, $n_{R}$, is higher than or equal to the number of transmitting ones, $n_{T}$, there exist suboptimum methods for LLR computation with near-optimum performance, such as ML or Maximum A Posteriori (MAP) List Sphere Detection (LSD) (Hochwald \& ten Brink, 2003; Vikalo et al., 2004) or Soft Interference Cancellation with MMSE filtering (SIC-MMSE) (Wang \& Poor, 1999). However, when $n_{T}>n_{R}$, these high-performance suboptimum detectors cannot be utilized. The reason is that either the underlying system of equations is underdetermined or the decoding complexity grows exponentially with $n_{T}-n_{R}$. To overcome this limitation, high data rate linear STBCs have been proposed under the name of Linear Dispersion (LD) codes (Hassibi \& Hochwald, 2002). In a sense, LD codes are an extension of V-BLAST for the case $n_{T}>n_{R}$. Since the use of LD codes modifies the EXIT characteristic of the resulting channel, it is necessary to specifically design codes matched to this new EXIT characteristic.

It is not clear in the literature which MIMO signaling scheme, i) concatenation of channel coding with an STBC or ii) BICM with spatial multiplexing, is better in terms of approaching capacity. For a $2 \times 1 \mathrm{MIMO}$ system, concatenation with the Alamouti code may appear preferable at a first glance since it employs simpler detectors and can approach capacity using conventional SISO optimized channel encoders. However, it is not clear whether this signaling technique is able to outperform BICM with spatial multiplexing when employing channel codes optimized for this specific MIMO configuration and each modulation format. For a $2 \times 2$ MIMO system, concatenating with a Golden code seems the best option to avoid the capacity loss introduced by the Alamouti code. Now, specific capacity approaching channel codes should be designed and, again, it is not known wether this concatenated scheme performs better than BICM with spatial multiplexing, or not. Finally, for a $3 \times 1 \mathrm{MIMO}$ system, either concatenation with a LD code or spatial multiplexing can be used. In each case, a specific channel code should be designed but the performance of these optimized coded modulation schemes is unknown 
In this chapter we shed light into this controversy by comparing the performance of the above-mentioned MIMO scenarios when SCLDGM capacity approaching codes are employed. The data rate is two bits per channel use for the $2 \times 1$ and the $2 \times 2$ cases, and one bit per channel use for the $3 \times 1$ scenario. We specifically optimized regular SCLDGM codes for each system configuration using EXIT analysis techniques and assess its ability to approach the MIMO channel theoretical capacity limits.

A major contribution of this chapter is that performance evaluation is carried out not only over synthetically generated, spatially uncorrelated, Rayleigh distributed, flat-fading channels (ergodic and quasi-static) but also over realistic indoor scenarios. Although computer simulations are necessary and recommendable for wireless systems evaluation, they only reflect the simulated environment rather than the actual scenarios in which wireless systems operate. Channel models typically omit important issues such as quantization effects, power amplifier non-linearities, mutual antenna coupling, and phase noise. This is particularly important when dealing with MIMO channels since the scientific community has not reached a consensus on a reference channel model due to the extremely large number of parameters to be considered.

For the experimental evaluation of the MIMO coded modulation techniques we have used a MIMO hardware demonstrator developed at the University of A Coruña. The demonstrator has been constructed from Commercial Off-The-Shelf (COTS) modules, including the RF front-ends. We also developed a distributed multilayer software architecture necessary for the configuration and utilization of the hardware platform. Different experiments were carried out at the $5 \mathrm{GHz}$ Industrial, Scientific and Medical (ISM) band considering different $\mathrm{Tx} / \mathrm{Rx}$ locations and antenna positions. The results are presented in terms of Block Error Rate (BLER) versus $E_{b} / N_{0}$ at reception and are representative of the performance obtained over a typical indoor scenario.

The remainder of this chapter is organized as follows: Section 2. describes the different MIMO signaling techniques under consideration, namely, BICM with spatial multiplexing or in concatenation with STBCs (Alamouti, Golden or LD codes). Section 3. explains the utilization of SCLDGM codes in the schemes under consideration, and the optimization procedure. Section 4. presents the results of computer simulations assuming an identically and independently distributed (i.i.d.), spatially white, flat-fading Rayleigh MIMO channel (ergodic and quasi-static). These computer simulations corroborate the validity of the designed codes and show that spatial multiplexing and concatenation with a STBC yield the same performance. Section 5.describes the hardware and the experiments carried out to assess the performance of the MIMO signaling methods over a realistic indoor scenario. The results confirm those obtained by simulations: the performance of systems employing BICM with spatial multiplexing is the same as that when concatenating with a STBC. Finally, Section 6. provides the conclusions of this study.

\section{Coded modulation for MIMO channels}

\subsection{Encoder}

Figure 1 shows the block diagrams of the two basic MIMO coded modulation signaling methods considered in this chapter: Bit Interleaved Coded Modulation (BICM) with spatial multiplexing and channel coding concatenated with a STBC. We will assume that a stream of information bits $\mathbf{u}=\left[u_{1}, u_{2}, \ldots, u_{K}\right]$ inputs a rate $R_{c}=K / N$ temporal encoder (in our case an SCLDGM encoder) to produce a coded bit sequence $\mathbf{c}=\left[c_{1}, c_{2}, \ldots, c_{N}\right]$. This sequence is 
then Gray-mapped to a constellation carrying $M_{\mathcal{c}}$ bits per symbol, resulting in the sequence $\mathbf{s}=\left[s_{1}, s_{2}, \ldots, s_{L}\right]$, with $L=N / M_{\mathcal{C}}$.

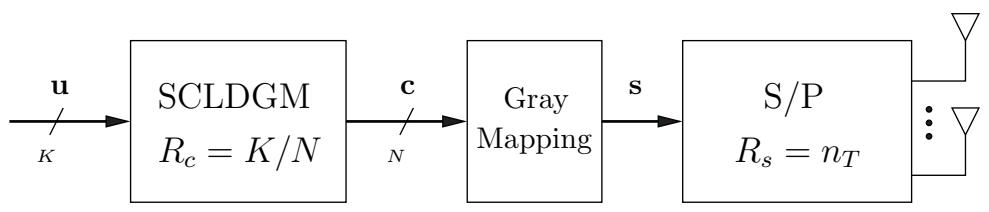

(a) BICM with spatial multiplexing

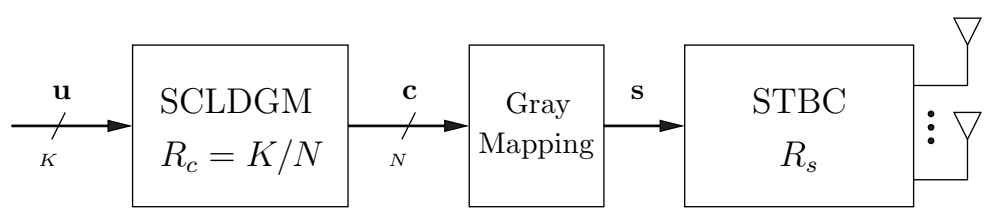

(b) Channel coding concatenated with STBC

Fig. 1. MIMO signaling schemes.

In the BICM scheme with spatial multiplexing (Fig. 1.a), the transmitted symbols $s_{k}$ are serial-to-parallel $(\mathrm{S} / \mathrm{P})$ converted to produce the sequence of transmitted vectors $\mathbf{s}[k], k=$ $1,2, \ldots, L / R_{S}$, where $R_{S}=n_{T}$ is the spatial rate. BICM with spatial multiplexing is a good option for signaling over a MIMO channel in the general case of $n_{T} \times n_{R}$ with $n_{T}, n_{R} \geq 2$, since, as we will see bellow, it is capable of approaching the capacity limits. However, the complexity of optimum MAP detection, exponential in both $n_{T}$ and $M_{c}$, constitutes an important limitation for a high number of transmitting antennas and/or modulation formats. In this case, it is possible to employ suboptimum detection methods such as LSD (List Sphere Detection) (Hochwald \& ten Brink, 2003; Vikalo et al., 2004) or SIC-MMSE (Soft Interference Cancellation with MMSE filtering) (Wang \& Poor, 1999). However, these methods either require the observations to be fully determined $\left(n_{T} \leq n_{R}\right)$ or have an exponential complexity in $n_{T}-n_{R}$. This is an important limitation because $n_{T}>n_{R}$ occurs frequently in practice (for example, in the downlink of cellular communication systems).

When an STBC is concatenated after the constellation mapper (Fig. 1.b), the sequence $\mathbf{s}$ is partitioned into blocks of $Q$ symbols. Each block is then encoded into an $n_{T} \times T$ symbol matrix, $\mathbf{S}[k]$, which is transmitted using $T$ channel uses, resulting in a spatial rate $R_{S}=Q / T$. The mapping $\left[s_{k}, s_{k+1}, \ldots, s_{k+Q}\right] \rightarrow \mathbf{S}[k]_{n_{T} \times T}$ performed by the STBC greatly affects the features of the MIMO system: it may change its associated capacity, the attained diversity, and the complexity of the detection process, as well as the applicable detection methods.

Notice that, in spite of their differences, the two MIMO signaling schemes in Fig. 1 are closely related. Indeed, BICM with spatial multiplexing can be interpreted as the concatenation of a temporal encoder and a trivial STBC with $Q=n_{T}$ and $T=1$.

\subsection{Channel model}

After transmission through the MIMO channel, the matrix of received vectors, $\mathbf{X}[k]$, is

$$
\mathbf{X}[k]=\mathbf{H}[k] \mathbf{S}[k]+\mathbf{N}[k], \quad k=1,2, \ldots, L / R_{s},
$$


where $\mathbf{H}[k]$ is the $n_{R} \times n_{T}$ MIMO channel matrix and $\mathbf{N}[k]=\left[\begin{array}{ll}\mathbf{n}_{1} & \mathbf{n}_{2} \cdots \mathbf{n}_{T}\end{array}\right]$, where each column $\mathbf{n}_{t}$ contains independent AWGN samples. For the simulations in Section 4. we will assume a spatially uncorrelated, Rayleigh-fading MIMO channel where the elements in $\mathbf{H}[k]$ are distributed as $\mathcal{C N}(0,1)$. Under the ergodic assumption, the channel matrix changes each time a new vector of symbols is transmitted, whereas under the quasi-static assumption, it remains constant during the transmission of a whole codeword (i.e., $\mathbf{H}[k] \equiv \mathbf{H}, k=$ $\left.1,2, \ldots, L / R_{S}\right)$. We assume in both cases that the channel changes in an independent fashion from one realization to the next.

For the experiments in Section 5, no assumption on the channel coefficients distribution is made. Since the delay spread in indoor channels is typically small, it is reasonable to assume that the flat-fading hypothesis holds true. Also, since neither the transmitter nor the receiver is moving, the channel behaves in a quasi-static manner and remains unchanged during the transmission of a data frame.

\subsection{Decoder}

In all the signaling methods we will assume that there is no Channel State Information (CSI) at the transmitter, while the receiver has perfect CSI. In the experiments in Section 5the channel is estimated in a previous step assuming that all transmitted symbols are known at the receiver, which yields to an almost perfect estimation of the channel.

Turbo-like receivers take as input the channel Log-Likelihood Ratios (LLRs), which have different expressions depending on the channel model and the detector type. For the sake of clarity, we will drop the time index $k$ hereafter.

For BICM with spatial multiplexing, the information contained in the received vector $\mathbf{x}$ about one of its bits, $v_{k}= \pm 1$, is represented by the channel LLR, $L_{\mathrm{ch}}$, which is computed by the optimum MIMO detector as

$$
\begin{aligned}
L_{\mathrm{ch}} & =\log \frac{P\left(\mathbf{x} \mid v_{k}=+1\right)}{P\left(\mathbf{x} \mid v_{k}=-1\right)}=\log \frac{P\left(v_{k}=+1 \mid \mathbf{x}\right)}{P\left(v_{k}=-1 \mid \mathbf{x}\right)}-\underbrace{\log \frac{P\left(v_{k}=+1\right)}{P\left(v_{k}=-1\right)}}_{L_{k}} \\
& =\log \frac{\sum_{\mathbf{s} \in \mathbf{S}_{k}^{+}} \exp \left(-\frac{\|\mathbf{x}-\mathbf{H s}\|^{2}}{N_{0}}+\sum_{i=1}^{n_{T} M_{c}} \frac{v_{i} L_{i}}{2}\right)}{\sum_{\mathbf{s} \in \mathbf{S}_{k}^{-}} \exp \left(-\frac{\|\mathbf{x}-\mathbf{H s}\|^{2}}{N_{0}}+\sum_{i=1}^{n_{T} M_{c}} \frac{v_{i} L_{i}}{2}\right)}-L_{k \prime}
\end{aligned}
$$

where $\mathbf{S}_{k}^{+}$and $\mathbf{S}_{k}^{-}$represent the set of all transmitted symbol vectors $\mathbf{s}$ where bit $v_{k}=+1$ and $v_{k}=-1$, respectively. Note that the MIMO detector makes use of the bit Log Prior Ratio (LPRs), $L_{k}$, which turn outs to be the output messages from the channel decoder.

When the Alamouti STBC is used concatenated with a channel encoder, optimum detection can be performed in an independent fashion over the transmitted streams, thanks to the orthogonality of the effective channel matrix. At the receiver, multiplying the observations by the Hermitian of the channel matrix gives two new observations, $x_{1}$ and $x_{2}$, corresponding to an equivalent, spatially decoupled model. Then, optimum computation of the channel LLRs for each stream, $x$, can be realized as 


$$
L_{\mathrm{ch}}=\log \frac{P\left(x \mid v_{k}=+1\right)}{P\left(x \mid v_{k}=-1\right)}=\log \frac{\sum_{s \in S_{k}^{+}} \exp \left(-\frac{\|x-\mathcal{F} s\|^{2}}{\mathcal{F} N_{0}}+\sum_{i=1}^{M_{c}} \frac{v_{i} L_{i}}{2}\right)}{\sum_{s \in S_{k}^{-}} \exp \left(-\frac{\|x-\mathcal{F} s\|^{2}}{\mathcal{F} N_{0}}+\sum_{i=1}^{M_{c}} \frac{v_{i} L_{i}}{2}\right)}-L_{k}
$$

where $\mathcal{F}=\|\mathbf{H}\|_{F}^{2}$ is the squared Frobenius norm of the channel matrix.

Optimum LLR computation for LD-coded MIMO systems can be carried out in a completely analogous way to (2) just by considering the resulting equivalent channel model. Regarding detection, the Golden code is also a linear code and, thus, the same equivalent observation model as that of linear dispersion codes applies. In any case, this different equivalent channel model has to be taken into account when performing code design.

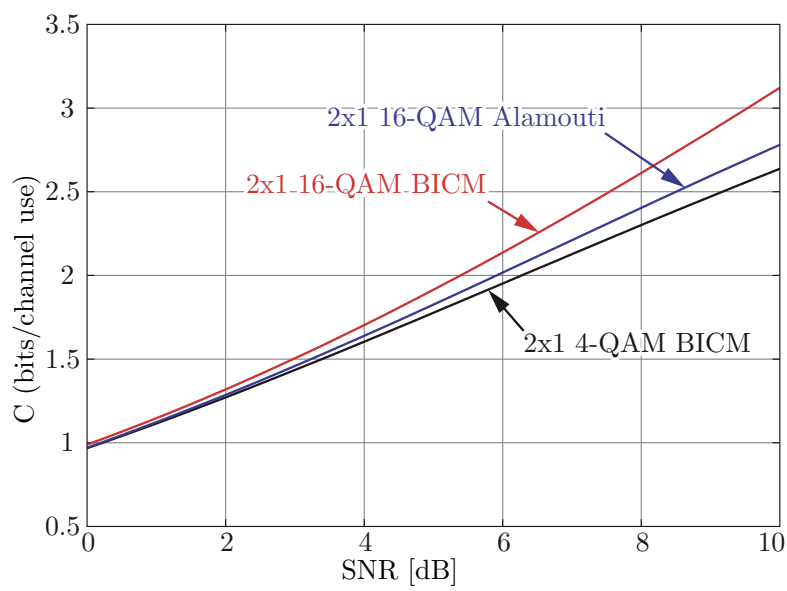

Fig. 2. Constrained capacity of $2 \times 1 \mathrm{BICM}$ with spatial multiplexing and in concatenation with Alamouti STBC in the region of interest.

\subsection{Constrained system capacity}

Figures 2, 3 and 5 plot the constrained capacities of the different MIMO coded modulation signaling schemes previously described for the cases of $2 \times 1$ (see Fig. 2), $2 \times 2$ (see Fig. 3) and $3 \times 1$ (see Fig. 5), respectively, over the spatially white, Rayleigh-distributed MIMO channel. The target rates are $2 \mathrm{bits} / \mathrm{channel}$ use for $2 \times 1$ and $2 \times 2$, and $1 \mathrm{bit} /$ channel use for $3 \times 1$. We use the term constrained capacity to refer to the channel capacity when the transmitter is constrained to use a specific modulation format (4-QAM, 16-QAM, etc.). This constrained capacity is calculated by measuring the mutual information between the output L-values from the detector, $L_{\mathrm{ch}}$, and their associated coded bits.

When $n_{T}=2$, the utilization of the Alamouti code as an inner code is very attractive because it allows for spatially decoupling the ML detection, notably simplifying the overall decoding procedure. The price to be paid is the spatial rate consumed by the Alamouti code, $R_{S}=1$, which forces the utilization of a higher order modulation to compensate for the rate loss (16-QAM for Alamouti versus 4-QAM in the BICM with spatial multiplexing 


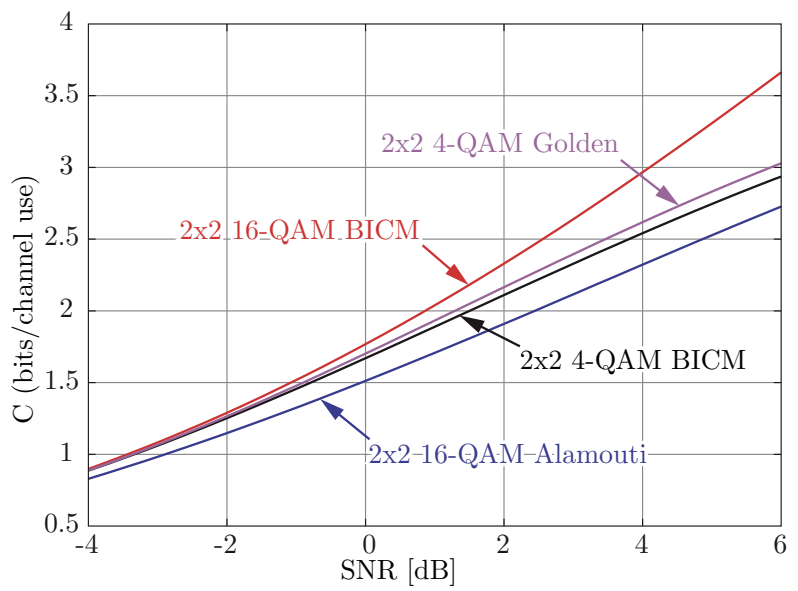

Fig. 3. Constrained capacity of $2 \times 2 \mathrm{BICM}$ with spatial multiplexing and in concatenation with STBCs (Alamouti and Golden) in the region of interest.

scheme). Moreover, the imposed signal structure degrades the capacity of the equivalent MIMO system: it is well known (Sandhu \& Paulraj, 2000) that the unconstrained capacity (i.e. with Gaussian input symbols) of a $2 \times n_{R}$ MIMO system with Alamouti coding is less than or equal to that of the MIMO channel without Alamouti coding. This is also true for the case of constrained capacity, as reflected in Figure 2 (top right) where the curve corresponding to 16-QAM Alamouti presents worse performance than that of 4-QAM BICM with spatial multiplexing. For the unconstrained capacity, the equality holds only for the case $n_{R}=1$ (Sandhu \& Paulraj, 2000). From Figure 2 (top left), it is clear that Alamouti coding is a good choice for $2 \times 1$ MIMO systems with constrained symbols, since in this case 16-QAM Alamouti outperforms 4-QAM BICM with spatial multiplexing.

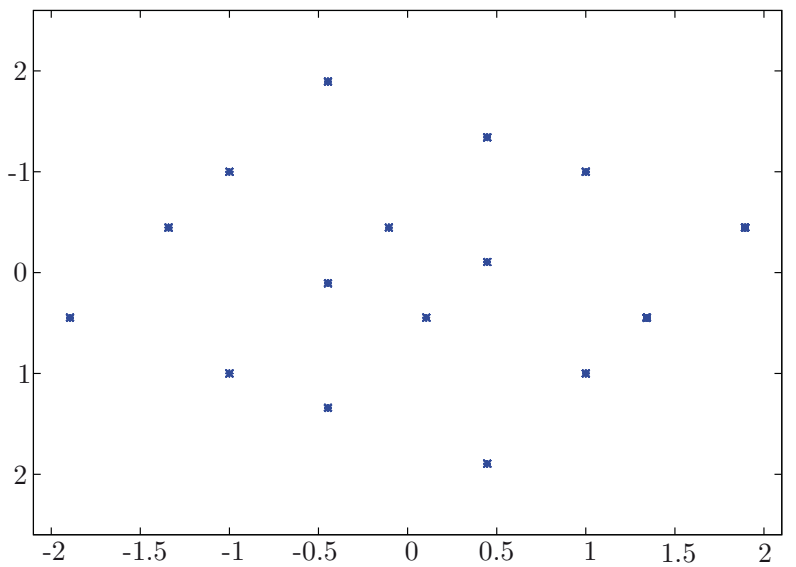

Fig. 4. Constellation at the output of the $2 \times 2$ Golden code when using a 4-QAM constellation at its input. 
When considering $2 \times 2$ MIMO systems, the Golden code is an appealing alternative to the Alamouti code. The Golden code (Belfiore et al., 2005) is a non-orthogonal $2 \times 2$ STBC with full information rate $\left(R_{S}=n_{T}=2\right)$ that provides a capacity improvement at a little increase in complexity, as it can be seen from Fig. 3. This capacity improvement is due to the fact that the resulting constellation at the output of the Golden encoder (see Fig. 4) resembles a Gaussian distribution better than the input constellation. This effect is usually referred to as shaping or constellation expansion (Forney, Jr. \& Wei, 1989). Although the size of the resulting constellation is larger, the Golden code does not introduce any redundancy because it employs two channel uses for the transmission of four input symbols.

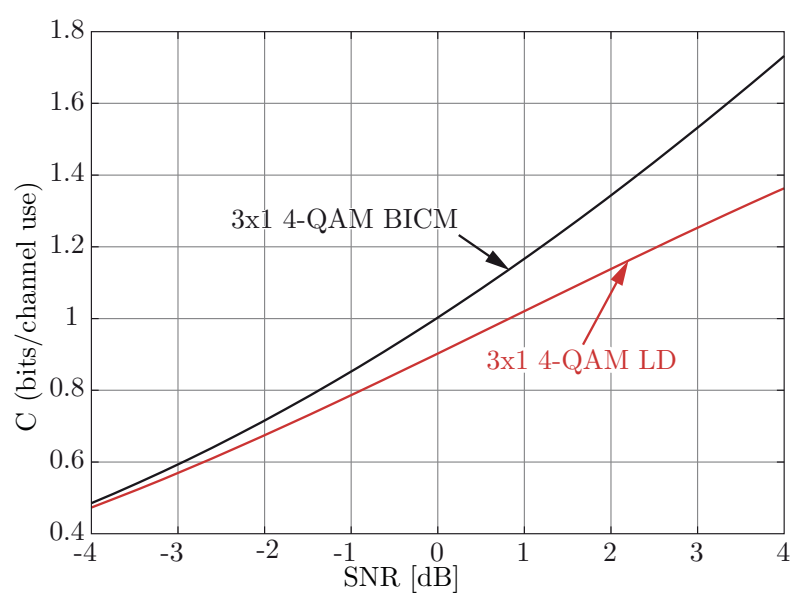

Fig. 5. Constrained capacity of $3 \times 1 \mathrm{BICM}$ with spatial multiplexing and in concatenation with LD STBC in the region of interest.

Finally, when $n_{T}>n_{R}$, the use of LD codes allows for the application of suboptimum detection methods without much capacity penalty. Linear Dispersion (LD) codes (Hassibi \& Hochwald, 2002) are linear STBCs that transform the observation model to avoid underdetermination, at the cost of a minimum capacity loss (see Fig. 5). A stacked, real-valued, equivalent observation model can then be easily formulated (Hassibi \& Hochwald, 2002). Such an equivalent observation model is not underdetermined provided that $n_{R} T>Q$ or, equivalently, $n_{R}>R_{s}$. Thus, concatenation with LD codes constitute a good choice when $n_{T}>n_{R}, n_{T}>2$, and optimum detection is not feasible. Notice also that, similar to the Golden code, constellation expansion takes place at the output of an LD encoder, but it is controlled because symbols are produced according only to specific sequences.

\section{Capacity approaching codes for MIMO transmission}

In order to approach the capacity of MIMO channels, both BICM with spatial multiplexing and schemes based on concatenation with STBCs have to use an appropriate channel code. In this chapter we focus on a particular subclass of LDPC codes known as Serially-Concatenated Low-Density Generator Matrix (SCLDGM) codes (Garcia-Frias \& Zhong, 2003), whose performance is similar to that of general LDPC codes but with very low encoding complexity. The convergence of any coding scheme can be predicted by tracking the mutual information of the different types of messages exchanged between the components of the receiver. This 
can be efficiently done by considering the EXtrinsic Information Transfer (EXIT) function (ten Brink, 1999) of each component. This procedure, termed EXIT evolution, has been successfully applied to obtain good SCLDGM codes for the Binary-Input AWGN (BIAWGN) channel (González-López et al., 2006a) and for MIMO BICM systems (Vázquez-Araújo et al., 2006; 2007).

The EXIT analysis is based on two assumptions. First, that each message can be expressed as the output LLR of a Binary-Input AWGN (BIAWGN) channel, which allows for the calculation of a bijection between the variance of the L-values and their associated mutual information, i.e., $I=J\left(\sigma^{2}\right)$ and $\sigma^{2}=J^{-1}(I)$ (ten Brink et al., 2004). Second, that the messages passed between the components are independent and identically distributed (i.i.d.). Under these two assumptions, the EXIT functions of SCLDGM codes (and, in general, of LDPC-based codes) can be easily calculated (González-López et al., 2007).

The EXIT function of the detector also needs to be calculated. Note that in BICM the channel LLRs produced by the optimum MIMO detector (see (2)), $L_{\mathrm{ch}}$, that constitute the input to the Turbo-like decoder, include the overall effect of the modulator, the channel and the detector. The EXIT function, $I_{\mathrm{ch}}\left(I_{A}, E_{b} / N_{0}\right)$, depends on the channel $E_{b} / N_{0}$ and the mutual information of the messages from the decoder, which constitutes the input a priori information to the detector, $I_{A}$. The characterization of the detector is independent of any other decoding module (ten Brink et al., 2004), so it can be obtained by measuring the mutual information of the $L_{\mathrm{ch}}$ messages in Monte Carlo simulations. It is important to highlight that any variation in the model parameters (modulation, number of transmit and receive antennas) leads to a different equivalent channel and thus to a different detector EXIT function. Consequently, there is a different optimum code for each antenna configuration and modulation format.

For Binary-Input AWGN (BIAWGN) channels, the detector EXIT function does not depend on the information from the decoder, because each bit is transmitted in an independent channel use. Furthermore, for Single-Input Single-Output (SISO) channels with perfect CSI at reception and the usual constellations (i.e., PSK and QAM), Gray mapping results in an overall effect of modulation, channel and demodulation with an associated EXIT function equivalent to a BIAWGN channel, that is, only dependent on $E_{b} / N_{0}$ (i.e., it is an horizontal line for each $E_{b} / N_{0}$ value) (Schreckenbach et al., 2003). Thus, optimal codes for BIAWGN are also optimum for SISO channels and for any modulation, provided Gray-mapping is used. As a corollary of this result, optimal codes for OSTBC-coded systems (in particular, Alamouti-coded) with Gray mapping are the same as those optimum for the BIAWGN channel.

For the case of LD codes (for which, in terms of detection, the Golden code is indeed a particular instance), the detector produces channel LLRs according to an equivalent channel model. In this case, the EXIT function of the detector is different from the one corresponding to BICM with spatial multiplexing over the same channel model. Consequently, optimum codes for LD-coded systems differ from those obtained for BICM with spatial multiplexing systems. Figure 6 plots the EXIT characteristic for $2 \times 2$ and $3 \times 1$ MIMO systems for either spatial multiplexing or LD coding. They correspond to SNR values at each receive antenna (equal to $n_{T} / N_{0}$ assuming each antenna radiates unit energy symbols) close to the convergence threshold of the best code found in each case. Let us first recall that the area property of an EXIT function states that the area below the curve equals the system capacity for a Binary Erasure Channel (BEC). This area property can be considered as approximate for the BIAWGN channel assumed for modelling the input and output messages of the MIMO detector. For a $2 \times 2$ system operating at $\mathrm{SNR}=2.0 \mathrm{~dB}$, it is apparent that the EXIT function corresponding to a Golden-coded system has a larger area below it than that corresponding to a BICM with 


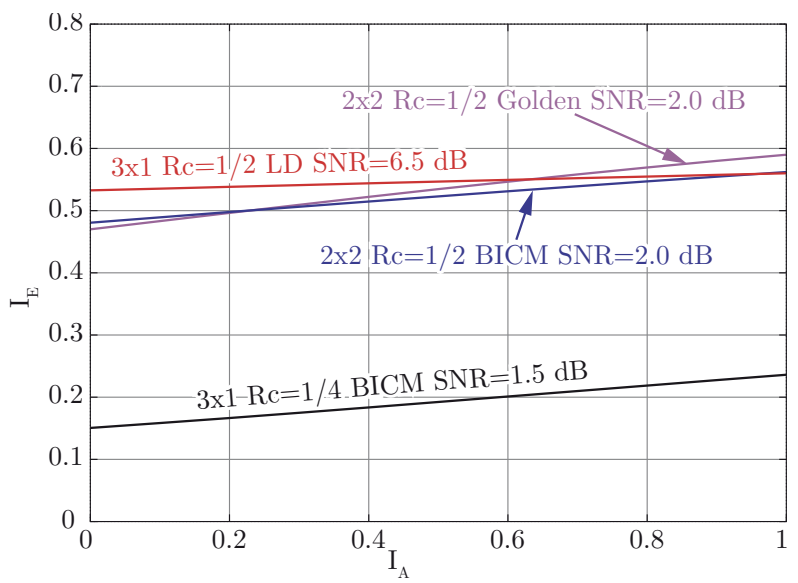

Fig. 6. EXIT characteristics corresponding to the considered MIMO schemes for a 4-QAM constellation. SNR is the signal-to-noise ratio at each receiving antenna (equal to $n_{T} / N_{0}$ assuming each antenna radiates unit energy symbols)

spatial multiplexing system, which is coherent with the capacity increase associated to the Golden code. Note also that the slope of the two functions is different, which leads to different optimum codes (cf. Table 1). For the $3 \times 1$ case, the EXIT function corresponding to BICM with spatial multiplexing is located far below the one corresponding to the LD-coded system, because the outer code rates are different $\left(R_{c}=1 / 6\right.$ and $1 / 2$, respectively) for the same overall information rate (1/6). Besides, their slopes are different. Both reasons justify that the optimum codes for these two schemes are very different from each other.

Once we have obtained the EXIT function of the detector and the decoder, system convergence can be tested by simulating the evolution of the mutual information through the iterations of both components. For a fixed $E_{b} / N_{0}$ we start with all information values equal to zero and, then, we iteratively compute their values. This is exactly what the decoding process does, except for using the associated information of the messages instead of their actual values. We say that the iterative receiver converges when we find a sequence of information values that finally leads to $I_{O}=1$, where $I_{0}$ is the mutual information associated to the a posterior $i$ L-values of the uncoded bits.

Table 1 presents the best regular SCLDGM codes obtained through EXIT analysis for the different MIMO signaling schemes. The table also shows the convergence thresholds predicted by this analysis, as well as the Constrained-input Capacity Limit (CCL) corresponding to each MIMO transmission method. We have considered the antenna configurations where concatenation with STBCs is more beneficial: $2 \times 1$ for the Alamouti code, $2 \times 2$ for the Golden code, and $3 \times 1$ for the LD code. In particular, the latter case presents the characteristics of being a clearly asymmetric antenna configuration $\left(n_{T}>n_{R}\right)$ as well as having a complexity low enough to appreciate the losses in i) capacity with respect to BICM with spatial multiplexing and ii) performance of suboptimum methods with respect to optimum detection in the LD-coded system. We have chosen these antenna configurations as our testbench to assess the gains provided by STBC-concatenated systems. For each antenna configuration, we optimize the SCLDGM code to maintain the overall information 


\begin{tabular}{|c|c|c|c|c|c|c|c|c|c|c|c|}
\hline Code & $n_{T} \times n_{R}$ & Modul. & STBC & $R_{c}$ & $p$ & $d_{u}^{f 1}$ & $d_{u}^{f 2}$ & $d_{p 1}^{f 2}$ & CCL & $\begin{array}{c}\text { Thresh } \\
(\mathrm{dB})\end{array}$ & $\begin{array}{c}\text { Gap } \\
(\mathrm{dB})\end{array}$ \\
\hline \hline$\# 1$ & $2 \times 1$ & 4 -QAM & None & $1 / 2$ & 0.0150 & 3 & 5 & 38 & 3.30 & 3.87 & 0.57 \\
$\# 2$ & $2 \times 1$ & 16 -QAM & None & $1 / 4$ & 0.0200 & 3 & 8 & 15 & 2.80 & 3.57 & 0.77 \\
\hline$\# 3$ & $2 \times n_{R}$ & Any & Alamouti & $1 / 2$ & 0.0200 & 3 & 6 & 6 & - & - & - \\
$\# 4$ & $2 \times n_{R}$ & Any & Alamouti & $1 / 4$ & 0.0400 & 3 & 9 & 24 & - & - & - \\
\hline$\# 5$ & $2 \times 2$ & $16-Q A M$ & None & $1 / 4$ & 0.0275 & 3 & 9 & 12 & 1.30 & 1.90 & 0.60 \\
$\# 6$ & $2 \times 2$ & 4-QAM & None & $1 / 2$ & 0.0300 & 3 & 5 & 32 & 1.56 & 2.02 & 0.46 \\
\hline$\# 7$ & $2 \times 2$ & 4-QAM & Golden & $1 / 2$ & 0.0250 & 3 & 5 & 38 & 1.42 & 1.87 & 0.45 \\
\hline$\# 8$ & $3 \times 1$ & 4-QAM & None & $1 / 6$ & 0.0350 & 3 & 10 & 24 & 0.40 & 1.20 & 0.80 \\
\hline$\# 9$ & $3 \times 1$ & 4-QAM & LD & $1 / 2$ & 0.0200 & 3 & 6 & 48 & 0.90 & 1.38 & 0.48 \\
\hline
\end{tabular}

Table 1. Optimized SCLDGM codes for MIMO channels. "Thresh" stands for the convergence threshold and "Gap" is the gap to the constrained-input capacity limit (CCL). When no STBC is employed, a BICM with spatial multiplexing scheme is considered.

rate fixed, so we can make a fair comparison between BICM with spatial multiplexing and the concatenated schemes.

As we will see in Section 4, SCLDGM codes optimized for Single-Input Single-Output (SISO) channels also exhibit good performance when used in concatenation with the Alamouti code in $2 \times n_{R}$ MIMO channels (González-López et al., 2006b). This is not a surprising result since the Alamouti code actually converts a $2 \times n_{R}$ MIMO channel into two parallel and independent SISO channels. Recall that the detector EXIT function of Gray-mapped SISO systems with all the standard constellations is an horizontal line (i.e. it is constant for any value of $I_{A}$ ), resulting in the same optimum code for any constellation. This explains why we do not specify the constellation and thus we cannot provide a threshold value for the Alamouti case, since this threshold is different depending on the employed modulation and the channel model.

The code design procedure described previously assumes an ergodic channel and an infinite block length. In (Yue et al., 2008) it is shown that, when optimizing for the quasi-static channel, codes optimized for the worst-case EXIT envelope can provide a better performance. In our case, however, the slope of the worst case envelope is very similar to that of the average EXIT curve, so the codes optimized for both cases are practically identical. Thus, we will restrict ourselves to ergodic optimization. We will show in Section 4.that the resulting codes present an excellent performance when used in quasi-static channels and/or with finite block lengths. Indeed, their gaps with respect to the outage limits when applied over quasi-static channels are very similar to the gaps they present in ergodic fast-fading channels.

\section{Simulation Results}

Computer simulations were carried out to illustrate the actual performance of the obtained SCLDGM coded modulation MIMO systems with data blocks of finite length over ergodic and quasi-static channels. For ergodic channels, the observed thresholds are slightly worse than those predicted, since EXIT function analysis assumes infinite-length data blocks. Note that the lower the code rate $\left(R_{c}\right)$ is, i) the higher the gap with respect to the Constrained-input Capacity Limit (CCL) for the best code found, and ii) the higher the gap between the theoretical threshold predicted by EXIT analysis and that observed in simulations. Similar 


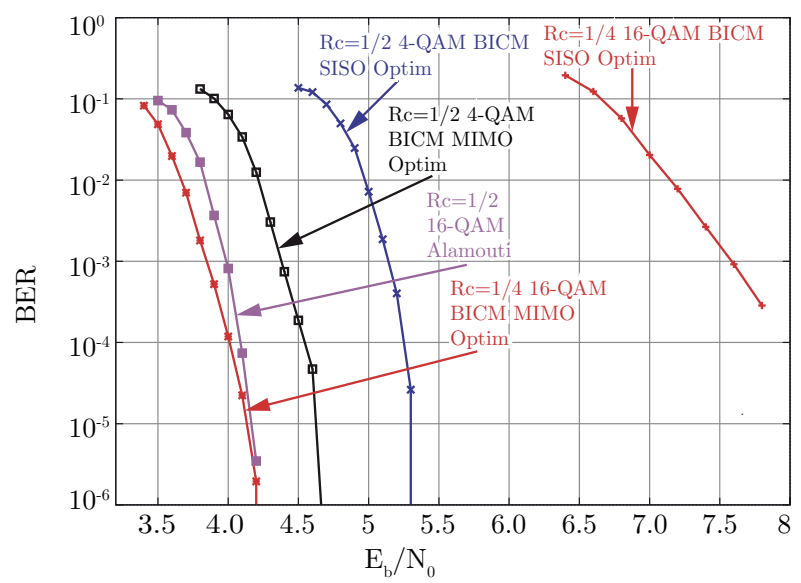

Fig. 7. Performance of a $2 \times 1 \mathrm{MIMO}$ system with a data rate of 2 information bits per channel use and i) SCLDGM + Alamouti code, ii) BICM with spatial multiplexing. Ergodic Rayleigh fading. The block length is $K=10,000$ information bits.

conclusions hold for quasi-static channels when comparing actual performance with respect to the outage probability limit.

\subsection{Ergodic channel}

Figure 7 shows the performance of several SCLDGM coded modulation $2 \times 1$ MIMO systems with a data rate of two bits per channel use assuming an ergodic channel model. The block length is $K=10,000$ information bits (we will also use this block length throughout all the simulations in ergodic channels) and 5,000 blocks are simulated for each $E_{b} / N_{0}$ value to obtain an adequate estimate of the Bit Error Rate (BER). The best performance is obtained when using BICM with spatial multiplexing, 16-QAM and code rate $1 / 4$ (code \#2). For a $\mathrm{BER}=10^{-4}$ (which we will use as the target BER from now on) the required $E_{b} / N_{0}$ is $4.0 \mathrm{~dB}$, which is $1.2 \mathrm{~dB}$ away from the CCL and $0.43 \mathrm{~dB}$ away from the theoretical threshold. Figure 7 also plots the performance obtained when using a concatenated Alamouti scheme with 16-QAM and code rate $R_{c}=1 / 2$, with the code optimized for the SISO channel (code $\# 3$ ). The SCLDGM rate was raised up to $R_{c}=1 / 2$ in order to maintain the data rate equal to two bits per channel use. Not surprisingly, the performance of these two schemes is very similar, because the capacity limit of a $2 \times 1$ system is the same irrespectively of whether Alamouti is used or not. Regarding receiver complexity, however, it is obvious that decoding in the concatenated scheme is considerably simpler.

The data rate of two bits per channel use can also be obtained using BICM with spatial multiplexing, 4-QAM and code rate $R_{c}=1 / 2$. Code \#1 has been specifically optimized for this particular situation and its performance is also shown in Fig. 7. Lowering the number of bits per symbol in the modulator, $M_{\mathcal{C}}$, is interesting because it yields to a considerable reduction of the detector complexity. At the target BER, the required $E_{b} / N_{0}$ is $4.55 \mathrm{~dB}(1.25 \mathrm{~dB}$ away from the CCL for 4-QAM and $0.63 \mathrm{~dB}$ away from the predicted threshold). Thus, this case maintains the same gap to the CCL as the 16-QAM case, but it exhibits a $0.5 \mathrm{~dB}$ performance degradation due to the capacity loss resulting from changing the modulation format (see Table 1). Figure 7 also illustrates the importance of designing SCLDGM codes for each specific 


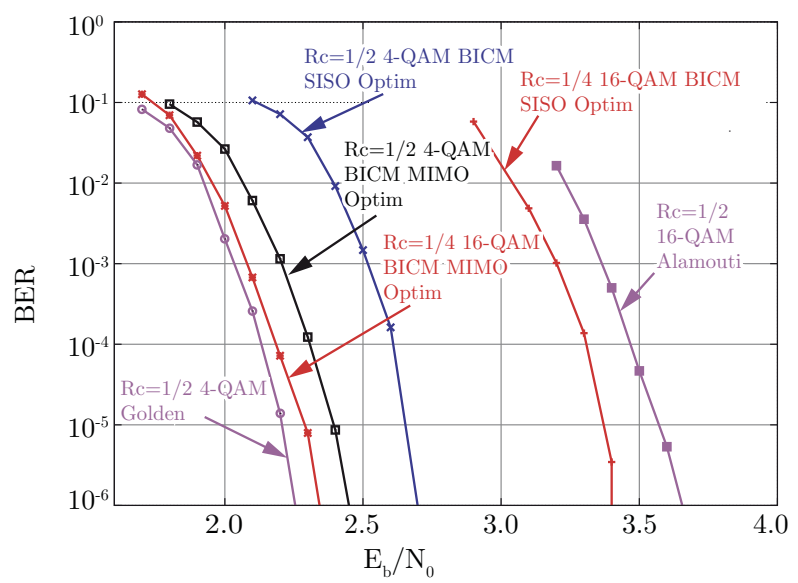

Fig. 8. Performance of a $2 \times 2 \mathrm{MIMO}$ system with a data rate of 2 information bits per channel use and i) SCLDGM + Golden code, ii) BICM with spatial multiplexing. Ergodic Rayleigh fading. The block length is $K=10,000$ information bits.

coded modulation MIMO configuration for BICM with spatial multiplexing. Indeed, observe the serious degradation in performance when the SISO-optimized codes \#3 and \#4 are used instead of the MIMO-optimized ones. In these cases, the required $E_{b} / N_{0}$ is 5.25 and $8.0 \mathrm{~dB}$, respectively, so that the loss in performance with respect to the MIMO-optimized codes is $0.7 \mathrm{~dB}$ and $4.0 \mathrm{~dB}$, respectively. From these results, we can conclude that code optimization is more critical when the constellation size increases.

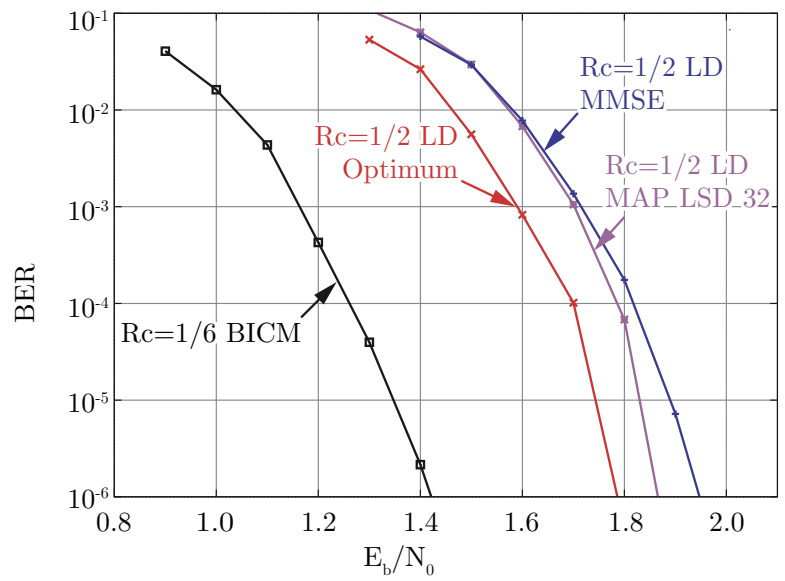

Fig. 9. Performance of a 4-QAM $3 \times 1$ MIMO system with a data rate of 1 information bit per channel use and i) SCLDGM $+R_{S}=1 / 3$ STBC (LD code), ii) BICM with spatial multiplexing. Ergodic Rayleigh fading. The block length is $K=10,000$ information bits.

The performance of a $2 \times 2$ MIMO system with a data rate of two bits per channel use is shown in Fig. 8 for an ergodic channel model and i) SCLDGM (code \#7) + Golden code, ii) BICM with spatial multiplexing (codes \#5 and \#6). Although the $R_{c}=1 / 416-\mathrm{QAM}$ BICM with spatial 
multiplexing system has the highest capacity (its corresponding CCL is at $E_{b} / N_{0}=1.30 \mathrm{~dB}$ ), the best performance is attained by the Golden-coded system (for which its corresponding CCL is at $E_{b} / N_{0}=1.43 \mathrm{~dB}$ ). This is explained because the best $R_{c}=1 / 4$ code found for the 16-QAM BICM with spatial multiplexing system has a threshold at $E_{b} / N_{0}=1.90 \mathrm{~dB}$ (theoretically) and at $E_{b} / N_{0}=2.20 \mathrm{~dB}$ (in practice), which is worse than that of the best $R_{c}=$ $1 / 2$ code found for the Golden-coded system (threshold at $E_{b} / N_{0}=1.87 \mathrm{~dB}$ theoretically and at $E_{b} / N_{0}=2.15 \mathrm{~dB}$ in practice). The $R_{c}=1 / 24$-QAM BICM with spatial multiplexing system shows worse performance, requiring $E_{b} / N_{0}=2.30 \mathrm{~dB}$ at the target $\mathrm{BER}$, which is consistent with its lower system capacity $\left(\mathrm{CCL}\right.$ at $\left.E_{b} / N_{0}=1.56 \mathrm{~dB}\right)$. We have also included the performance obtained for these systems when the code is the optimum for a SISO model (González-López et al., 2006a). The gaps in performance with respect to the MIMO-optimized codes are significant, especially for 16-QAM ( $0.3 \mathrm{~dB}$ for 4-QAM and $1.10 \mathrm{~dB}$ for 16-QAM). Finally, observe the poor performance of the Alamouti coded system $(1.25 \mathrm{~dB}$ worse than the MIMO-optimized code) even when using its optimum code (González-López et al., 2006b), which is a consequence of its system capacity loss.

Figure 9 shows the results for a $3 \times 1$ ergodic MIMO channel when using 4-QAM with a data rate of one information bit per channel use and i) SCLDGM (code \#9) + LD code, ii) BICM with spatial multiplexing (code \#8). The best performance is exhibited by the BICM with spatial multiplexing system employing an $R_{c}=1 / 6$ SCLDGM code (which requires an $E_{b} / N_{0}$ of $1.25 \mathrm{~dB}$ for the target BER). As explained before, using the LD code given by (36) in (Hassibi \& Hochwald, 2002) enables the application of suboptimum schemes such as LSD or SIC-MMSE at the cost of sacrificing capacity and, thus, performance. The degradation in actual performance (under optimum detection) with respect to BICM with spatial multiplexing is not severe $(0.45 \mathrm{~dB}$ at the target BER) and is of the same order as the loss in capacity (the CCL for BICM with spatial multiplexing is at $E_{b} / N_{0}=0.40 \mathrm{~dB}$ whereas for the LD code it is at $E_{b} / N_{0}=0.90 \mathrm{~dB}$ ). In addition, when the LD code is used, the gap of either MAP LSD or SIC-MMSE with respect to optimum detection is fairly small (around $0.1 \mathrm{~dB}$ ). This corroborates the convenience of employing LD coding as a means of enabling suboptimum detection methods when receiver complexity is a constraint.

\subsection{Quasi-static channel}

We have also studied the performance of SCLDGM coded modulation MIMO schemes when transmitting over quasi-static channels. We employ the same codes as in the ergodic channel, aiming at assessing if optimization for fast fading also leads to good performance in quasi-static scenarios $^{1}$. Figure 10 shows the performance of an SCLDGM BICM with spatial multiplexing scheme and an SCLDGM + Alamouti scheme over a $2 \times 1$ quasi-static MIMO channel. The channel block length is $B=500$ symbol vectors, which corresponds to $K=1000$ information bits. As it occurs in the ergodic channel, the performance of the Alamouti scheme and the BICM with spatial multiplexing and 16-QAM scheme are practically identical. The gap to the outage capacity is approximately $1.0 \mathrm{~dB}$ in both cases. The figure shows that the SCLDGM coded modulation scheme achieves maximum diversity, since the slope of the curve is the same as that of the outage capacity.

On the contrary, it is apparent from Fig. 10 that BICM with spatial multiplexing and 4-QAM performs worse $\left(5 \mathrm{~dB}\right.$ at a BLER of $\left.10^{-2}\right)$ than the other two methods. Notice the lower slope

\footnotetext{
${ }^{1}$ Notice that optimizing for short quasi-static channels would require the development of a completely different approach, which could be skipped if the codes optimized for ergodic, fast-fading behave well in quasi-static environments.
} 


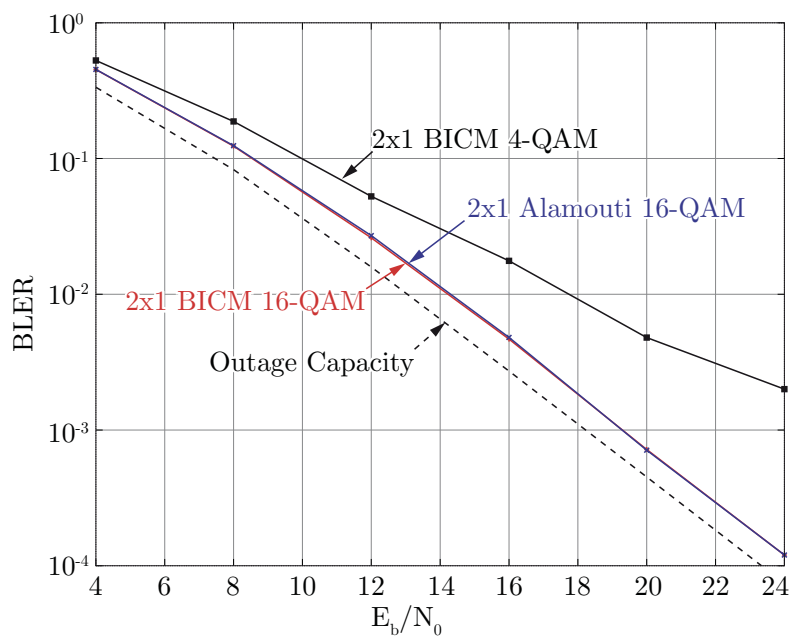

Fig. 10. Performance of a 16-QAM $2 \times 1$ MIMO system with a data rate of 2 information bits per channel use and i) BICM with spatial multiplexing and ii) SCLDGM + Alamouti. Quasi-static Rayleigh fading. The block length is $B=500$ symbol vectors.

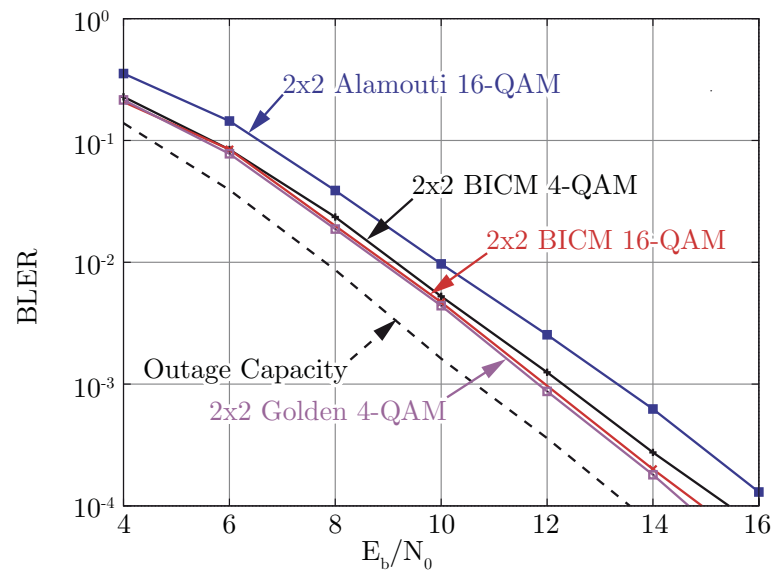

Fig. 11. Performance of a $2 \times 2$ MIMO system with a data rate of 2 information bits per channel use and i) BICM with spatial multiplexing, ii) SCLDGM + Golden code, iii) SCLDGM + Alamouti. Quasi-static Rayleigh fading. The block length is $B=500$ symbol vectors.

of the BLER curve for BICM with spatial multiplexing and 4-QAM, which means that this system is not able to extract all the spatial diversity available in the channel. We conjecture two explanations for this: on the one hand, it may happen that the high rate of the channel encoder $\left(R_{c}=1 / 2\right)$ and its subsequent mapping into 4-QAM symbols does not introduce enough redundancy for the signaling scheme to obtain all the available spatial diversity; on the other hand, the degree profile of the channel encoder has been designed assuming an ergodic channel and now the channel is quasi-static. 


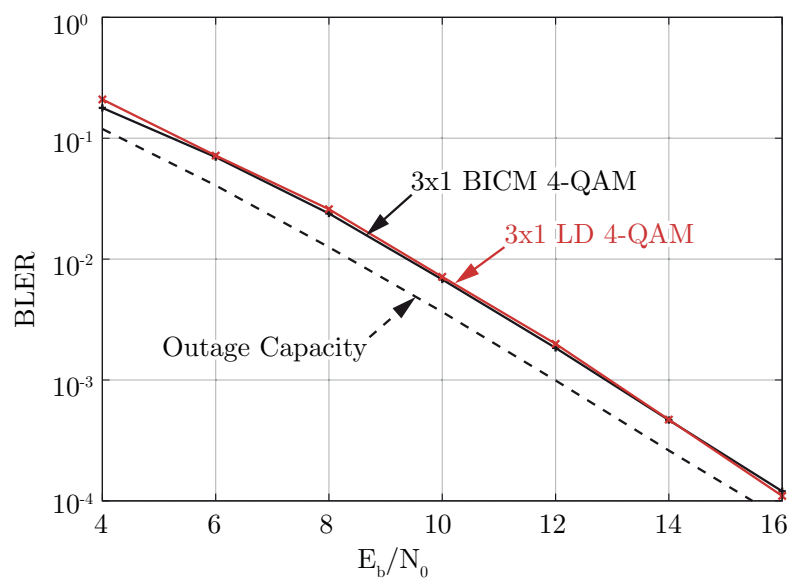

Fig. 12. Performance of a 4-QAM $3 \times 1$ MIMO system with a data rate of 1 information bit per channel use and i) SCLDGM $+R_{S}=1 / 3$ STBC (LD code), ii) BICM with spatial multiplexing. Quasi-static Rayleigh fading. The block length is $B=500$ symbol vectors.

Figure 11 shows the performance of an SCLDGM BICM with spatial multiplexing scheme, an SCLDGM + Golden code scheme and an SCLDGM + Alamouti scheme over a $2 \times 2$ MIMO channel. The best performance is achieved by the SCLDGM + Golden code, although the difference with the 16-QAM BICM with spatial multiplexing scheme is minimal. The gap to the outage capacity is kept at approximately $1.0 \mathrm{~dB}$ for the Golden and the BICM with spatial multiplexing with 16-QAM schemes and a little over $1.5 \mathrm{~dB}$ for the BICM with spatial multiplexing 4-QAM scheme. The distance of the Alamouti scheme to the outage capacity increases to $3.0 \mathrm{~dB}$. Again, all SCLDGM coded modulation schemes achieve maximum diversity. This demonstrates that the BICM with spatial multiplexing scheme is also suitable for quasi-static channels, without having to resort to schemes that explicitly maximize the spatial diversity. Similar results can be observed in Fig. 12 for a quasi-static $3 \times 1$ MIMO channel with $B=500$ symbol vectors ( $K=500$ information bits). The BICM with spatial multiplexing scheme is able to achieve the same diversity as the LD scheme, and both have very similar performance (approximately $1.0 \mathrm{~dB}$ away from the outage capacity).

\section{Experimental Evaluation}

For the experimental evaluation of the aforementioned schemes in a realistic indoor environment we employed a testbed developed at the University of A Coruña (García-Naya et al., 2010). A picture of the testbed is shown in Fig. 13. The testbed has been constructed using Commercial-Off-The-Shelf (COTS) modules from Sundance Multiprocessor (Sundance Multiprocessor, 2010) for the implementation of the baseband functionalities, and Radio Frequency (RF) font-ends from Lyrtech (Lyrtech, 2010). The hardware of the testbed is completed with a distributed, multilayer software architecture specifically designed to easy the interaction with the testbed hardware (Fernández-Caramés et al., 2008; García-Naya et al., 2010; García-Naya et al., 2008).

Figure 14 shows a block diagram containing the software and hardware elements utilized at the transmit side to assess the aforementioned schemes. Once the discrete-time, 


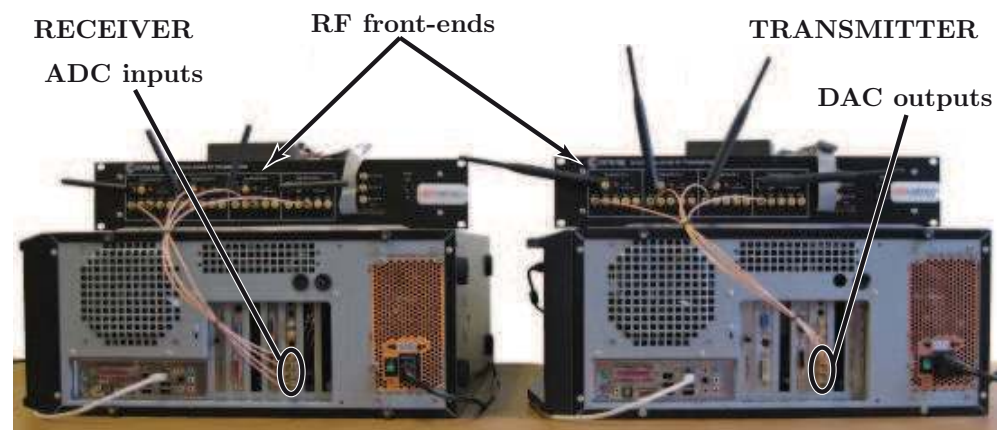

Fig. 13. Picture of the testbed developed at the University of A Coruña.

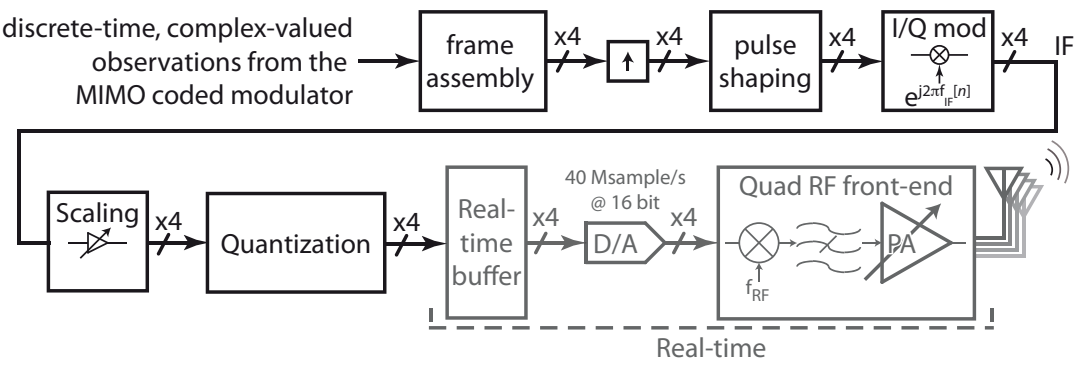

Fig. 14. Block diagram of hardware and software elements at the transmitter. Notice that depending on the scheme, two or three of the four transmit antennas are utilized.

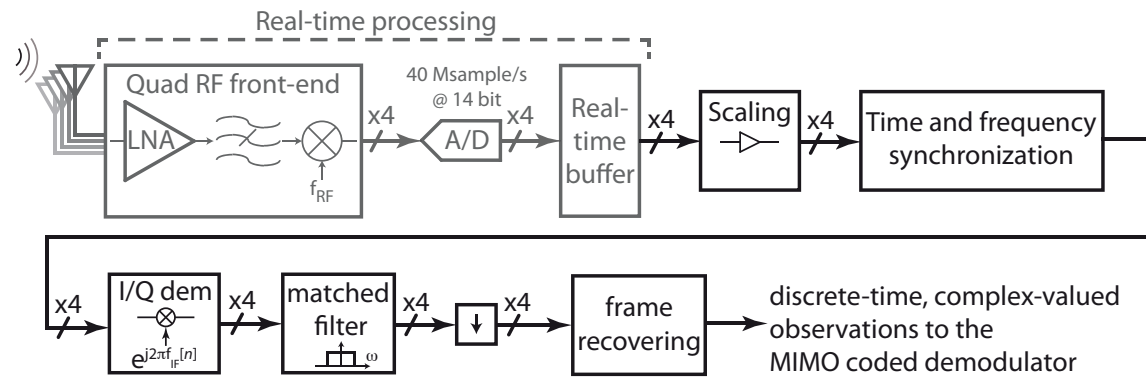

Fig. 15. Block diagram of hardware and software elements at the receiver. Notice that at the receiver, all four antennas are always utilized regarding of the scheme being acquired. Later, during the evaluation step, the corresponding signals are employed according to the scheme.

complex-valued source symbols from the encoder are generated, the following steps are carried out:

- The transmit frame is assembled. Basically, a preamble for time and frequency synchronization as well as a small silence for estimating the power spectral density of the noise are included. Then, for each transmit antenna (two or three depending on the scheme): 
- Up-sampling by a factor of 15 , resulting in 15 samples per symbol.

- Pulse-shape filtering using a squared root-raised cosine filter with $12 \%$ roll-off. Consequently, given that the sampling frequency of the DACs is set to $40 \mathrm{MHz}$, then the resulting signal has a bandwidth of $2.9867 \mathrm{MHz}$, which leads - according to our tests - to a frequency-flat channel response.

- The resulting signals are I/Q modulated to obtain a passband signal at a carrier frequency of $5 \mathrm{MHz}$.

- Such signals are then properly scaled in order to guarantee that the same transmit power level is achieved.

- Given the 16 bits of resolution of the DAC, the signals are properly quantized, obtaining 16-bit integer values for the samples.

- The resulting signal is then stored off-line in the buffer available at the hardware testbed.

- When the transmitter is triggered, such a buffer is read cyclically and in real-time by the DAC, which generates a signal at the intermediate frequency of $5 \mathrm{MHz}$.

- The resulting analog signal is sent to the RF front-end to be transmitted at the desired RF center frequency. In our measurements we utilized 69 different carriers in the frequencies ranging from $5200 \mathrm{MHz}$ to $5250 \mathrm{MHz}$ and from $5480 \mathrm{MHz}$ to $5700 \mathrm{MHz}$.

At the receiver side, once the transmitter has been triggered the following steps are carried out (see Fig. 15)

- The RF front-end down-converts the signal received by the four available antennas to the $5 \mathrm{MHz}$ intermediate frequency.

- The signal is then digitized by the ADCs and, in real-time, stored in the buffer. Given that the signals are being transmitted cyclically and in order to guarantee that a whole frame is acquired, twice the length of the transmit frame is acquired.

- The signals are properly scaled according to the number of resolution bits of the ADC. Notice that this factor is constant during the whole measurement campaign, thus not affecting the properties of the channel.

- In the next step, time and frequency synchronization operations are carried out.

- I/Q demodulation and filtering tasks take place. As a result, discrete-time, complex-valued observations with 15 samples per symbol are obtained. With the resulting samples (prior to the decimation stage) the instantaneous receive power as well as the instantaneous power spectral density of the noise are estimated. During the evaluation stage, using all instantaneous values estimated, the mean signal-to-noise ratio (SNR) is estimated.

- After filtering, the resulting signals are decimated, resulting in a single sample per symbol.

- Finally, the frame is properly disassembled, and the resulting observations are then sent to the MIMO coded demodulator.

\subsection{Measurement Procedure}

We evaluated experimentally the performance of the aforementioned MIMO schemes in a typical indoor environment (the research lab where the authors work at University of A Coruna) with a separation between the transmitter and the receiver about $9 \mathrm{~m}$, employing monopole antennas both at the transmitter and at the receiver. The antenna spacing is set 


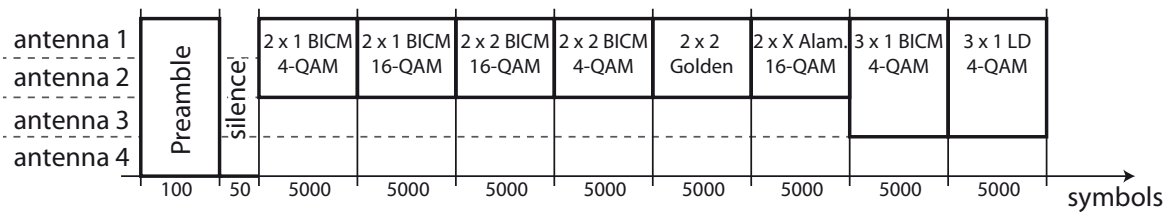

Fig. 16. Frame structure employed in the experimental evaluation.

to approximately 7 centimeters (determined by the separation of the antenna ports of the RF front-end). We experimentally observed that the channel behaved as non time-dispersive when transmitting with a bandwidth of $2.9867 \mathrm{MHz}$. This is an expected behavior since the delay spread of wireless channels in indoor environments is usually small.

We designed the frame structure shown in Fig. 16 in which, at the beginning, a pseudo random BPSK sequence (duration: 100 microseconds $^{2}$ ) is used as a preamble for subsequent time and frequency synchronization at the receiver. Next, a silence is introduced (duration: 19 microseconds) with the objective of estimating the power spectral density of the noise at the receiver. Next, the eight blocks corresponding to the eight different schemes to be evaluated are transmitted. Each block occupies 5000 symbols, resulting in a duration of 1.875 milliseconds. In total, the frame takes approximately 15 milliseconds for the transmission.

Neither the transmitter nor the receiver were moving during the transmissions. Also, experiments were carried out in a controlled scenario (at night) with no moving objects in the surroundings. This ensured the channel remained unchanged during the transmission of all the symbols corresponding to a frame containing the eight blocks.

Given that we know all transmit symbols and that we always measure in the high SNR regime (SNR > $20 \mathrm{~dB}$ ), we utilize all observations to perform a highly-accurate channel estimation. Additionally, we checked if the channel actually changed from the first to the last of the eight blocks of the frame and we verified that the channel remained constant during the transmission of the whole frame. Consequently, all eight schemes experience the same channel realization, thus ensuring a fair comparison among them.

In summary, experiments were carefully designed to ensure that the channel is frequency-flat and quasi-static. This is crucial to ensure the proper performance of the decoding algorithms. In order to simplify the evaluation stage, we always transmit at high transmit power level, avoiding non-linear effects caused by the saturation of the power amplifiers and, at the same time, guaranteeing an SNR value above $20 \mathrm{~dB}$. This, on the one hand, ensures that the errors in the synchronization will not cause a significant impact on the observed results. On the other hand, the estimates of the channel will be accurate enough to be able to evaluate the results assuming perfect CSI at the receiver, thus not including the effects of the channel estimation in the results.

With the aim of obtaining different channel realizations, we make use of the following three techniques:

- Given that the Lyrtech RF front-end is frequency-agile, we measure at different RF carriers in the interval ranging from $5200 \mathrm{MHz}$ to $5250 \mathrm{MHz}$ and from $5480 \mathrm{MHz}$ to $5700 \mathrm{MHz}$ and spaced $4 \mathrm{MHz}$ (greater than the bandwidth occupied by the signal), which results in 69 different frequencies, providing 69 different channel realizations.

\footnotetext{
2 the preamble is sampled at 40 samples per symbol, resulting in $1.12 \mathrm{MHz}$ of bandwidth
} 
- For all 69 different frequencies, we measure at 7 different antenna positions. Such positions are obtaining by moving the receiver in a distance in the order of a wavelength.

- Finally, given that none of the evaluated schemes makes use of all four receive antennas, we utilize each set of receive antennas because they experience a different channel realization. For example, when a $2 \times 1$ scheme is being evaluated, we obtain four different channel realizations, one at each receive antenna. Similarly, when a $2 \times 2$ scheme is being evaluated, two different channel realizations are obtained.

After all, depending on the number of receive antennas we obtain a different number of channel realizations. In the set-ups that only use a single receive antenna, we have $69 \times 7 \times 4=$ 1932 different channel realizations. In the set-ups that make use of two receive antennas, $69 \times 7 \times 2=966$ different channel realizations are available.

It is interesting to examine the statistical properties of the measured channels. Figure 17 plots the histograms of the module and the angle of the estimated channel coefficients for the $2 \times 2$ measurements. It is clear from these histograms that the magnitude and phase of the measured wireless channel coefficients match quite well a Rayleigh and a uniform distribution, respectively. Additionally, it is important to look at the spatial correlation among the channel coefficients. Towards this aim we stacked in a single $4 \times 1$ vector $\mathbf{h}$ the four coefficients of the $2 \times 2$ MIMO channel measured, i.e.,

$$
\mathbf{h}=\left[\begin{array}{l}
h_{11} \\
h_{12} \\
h_{21} \\
h_{22}
\end{array}\right],
$$

where $h_{i j}$ represents the complex-valued channel coefficient between the $j$-th transmit antenna and the $i$-th receive antenna. The covariance matrix of this vector is $\mathbf{C}_{\mathbf{h}}=\mathrm{E}\left[\mathbf{h} \mathbf{h}^{H}\right]$, where $\mathrm{E}[\cdot]$ denotes the expectation operator and $(\cdot)^{H}$ represents the conjugate transpose operation. From the measured channel coefficients we estimated this covariance matrix and we obtained the following matrix for their absolute values

$$
\mathbf{C}_{\mathbf{h}}=\left[\begin{array}{llll}
1.028395 & 0.018719 & 0.013984 & 0.028464 \\
0.018719 & 1.156758 & 0.022994 & 0.018275 \\
0.013984 & 0.022994 & 0.591004 & 0.029860 \\
0.028464 & 0.018275 & 0.029860 & 0.768709
\end{array}\right]
$$

Notice from (5) the low values of the non-diagonal elements which indicates that experiments were carried out in a rich scattering environment that introduced a low spatial correlation among the channel coefficients.

In summary, the statistical properties of the measured channel are very similar to those of the quasi-static channel considered in section Section 4.2

\subsection{Experimental Results}

Once the experiments were carried out and the received signals were recorded, experimental evaluation of the MIMO coded modulation methods was done in terms of Block Error Rate (BLER) versus $E_{b} / N_{0}$. Since the transmit power and the receiver noise level were fixed during each experiment, simulated AWGN was injected into the recorded received signals to change the operating $E_{b} / N_{0}$ value. Although the measurements were carried out at high SNR values 

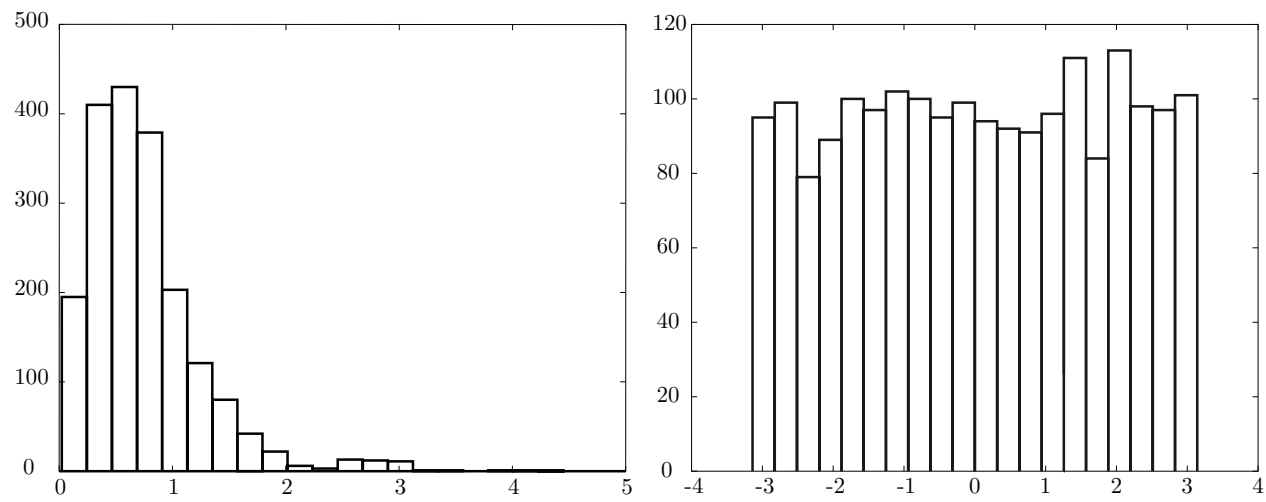

Fig. 17. Histogram of the module (left-hand) and the angle (right-hand) of the estimated channel coefficients for the $2 \times 2$ case.

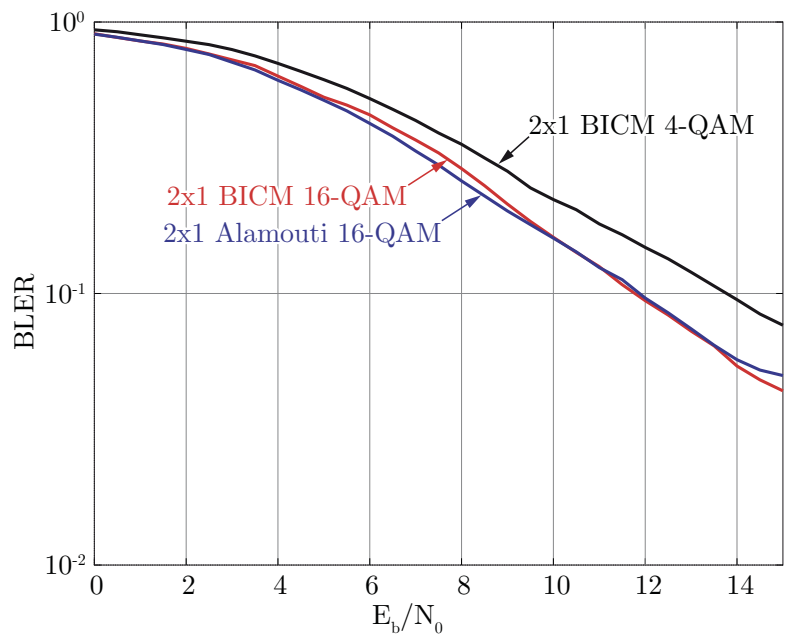

Fig. 18. Testbed results of $2 \times 1$ BICM with spatial multiplexing and in concatenation with STBCs.

and the target SNR is much lower than that of the measurements, we estimate the mean noise power level of the measurements as it was explained above and we take it into account when calculated the noise power level to be added in order to obtain the target mean $E_{b} / N_{0}$.

Figure 18 shows the experimental performance of the SCLDGM MIMO coded modulation systems for the $2 \times 1$ configuration and a data rate of two bits/channel use. Notice from this figure that the performance of BICM with spatial multiplexing and 16-QAM is practically the same as concatenating with the Alamouti code. This is in accordance with the constrained capacity analysis in Section 2.4and the simulation results in Section 4.2that also showed that both methods perform the same. On the contrary, it is apparent from Fig. 18 that BICM with spatial multiplexing and 4-QAM performs worse ( $2 \mathrm{~dB}$ at a BLER of $\left.10^{-1}\right)$ than the other 


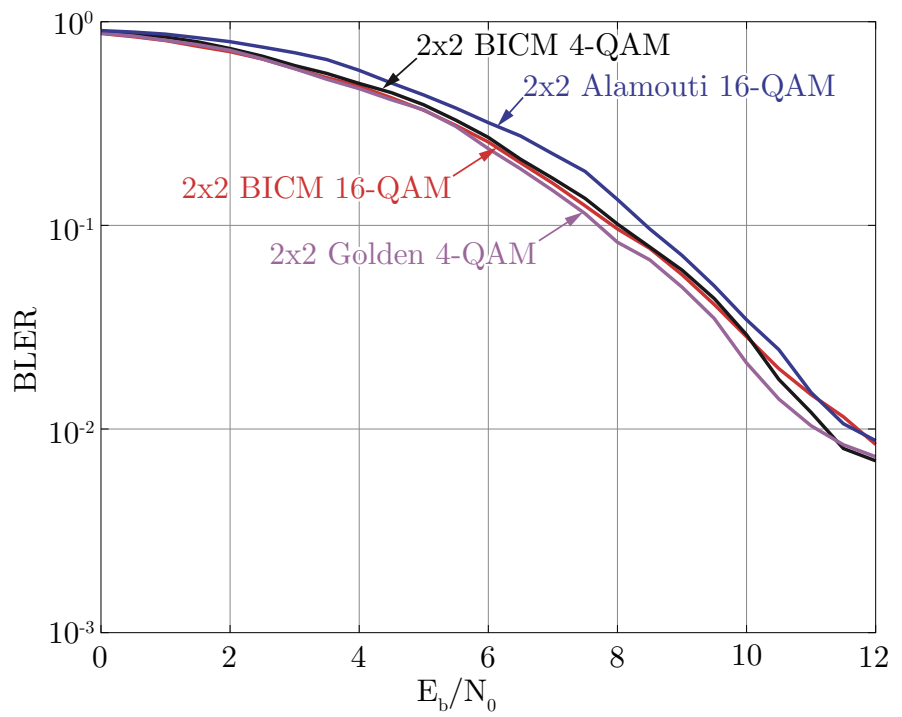

Fig. 19. Testbed results of $2 \times 2$ BICM with spatial multiplexing and in concatenation with STBCs.

two methods. This performance degradation was also appreciated during the simulations over a quasi-static channel (see Fig. 10). Notice the lower slope of the BLER curve for BICM with spatial multiplexing and 4-QAM, which means that this system is not able to extract all the spatial diversity available in the channel. We conjecture two explanations for this: on the one hand, it may happen that the high rate of the channel encoder $\left(R_{c}=1 / 2\right)$ and its subsequent mapping into 4-QAM symbols does not introduce enough redundancy for the signaling scheme to obtain all the available spatial diversity; on the other hand, the degree profile of the channel encoder has been designed assuming an ergodic, spatially white channel and these hypotheses are no longer true when dealing with the experimental channels.

Figure 19 shows the experimental performance of the SCLDGM MIMO coded modulation systems for the $2 \times 2$ configuration and a data rate of two bits/channel use. First of all, notice that the slope of all the BLER curves is twice larger than that of Fig. 18. This is because the four MIMO coded modulation methods are obtaining the full $n_{T} \times n_{R}=4$ spatial diversity of the channel. Next, notice that from a practical point of view, the performance difference among the methods is negligible. This is in accordance with the capacity analysis in Section 2.4and the quasi-static channel simulation results in Section 4.2that also showed that the performance difference among all methods is less than $1 \mathrm{~dB}$.

Finally, Fig. 20 shows the experimental performance of the SCLDGM MIMO coded modulation systems for the $3 \times 1$ configuration and a data rate of one bit/channel use. Again, note that the slope of the BLER curves is less than that of Fig. 19 but larger than than of Fig. 18 because the spatial diversity of a $3 \times 1 \mathrm{MIMO}$ channel is less than that of a $2 \times 2 \mathrm{MIMO}$ channel but larger than that of a $2 \times 1 \mathrm{MIMO}$ channel. Also note that the performance of the BICM with spatial multiplexing scheme is the same as that of the scheme concatenated with a LD code as expected from the capacity analysis in Section 2.4and the quasi-static channel simulations in Section 4.2 


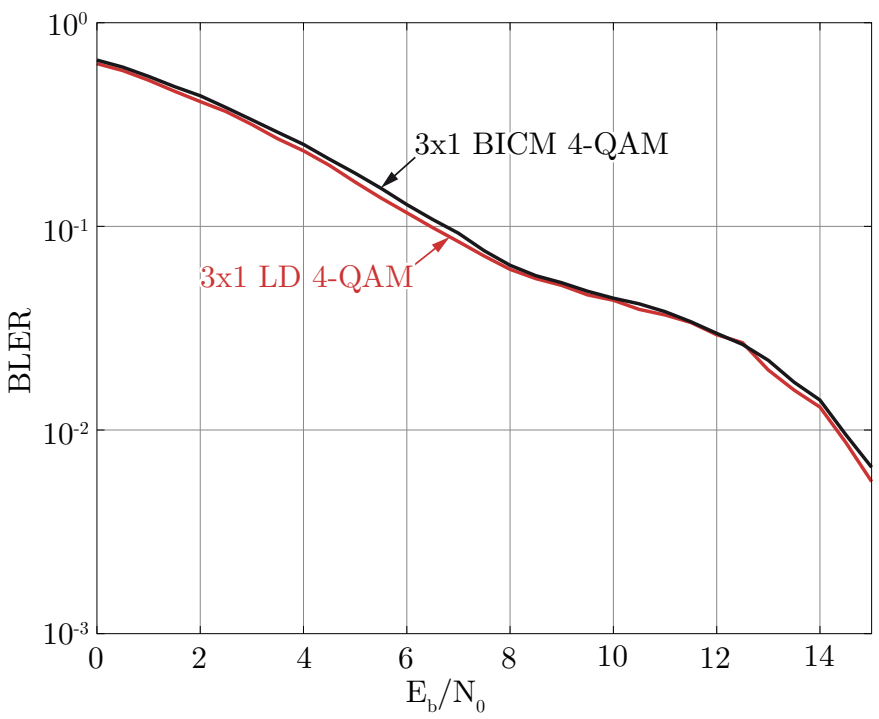

Fig. 20. Testbed results of $3 \times 1 \mathrm{BICM}$ with spatial multiplexing and in concatenation with STBCs.

\section{Conclusion}

We have studied MIMO coded modulation transmission schemes using either BICM with spatial multiplexing or concatenation of channel code with an STBC code, assuming turbo-like decoding at reception. Our study aims to shed light at the controversy on which of the two schemes is better to approach the capacity of MIMO channels. We have restricted ourselves to the less complex scenarios: $2 \times 1$ and two bits/channel use; $2 \times 2$ and two bits/channel use; and $3 \times 1$ and one bit per channel use. When using BICM with spatial diversity and two transmit antennas, these target data rates can be achieved with either a rate $1 / 2$ channel encoder and 4-QAM, or with a rate 1/4 channel encoder and 16-QAM. With three transmit antennas, the BICM with spatial multiplexing uses a rate $1 / 6$ channel encoder and 4-QAM. For the alternative coded modulation methods, the STBCs that were considered are: the Alamouti code for $2 \times 1$ and $2 \times 2$ configurations; the Golden code for $2 \times 2$; and the Linear Dispersion (LD) code for $3 \times 1$.

We have explained how to design regular SCLDGM codes for each specific MIMO coded modulation system. Code optimization has been carried out for spatially white flat-fading Rayleigh ergodic channels. By means of computer simulations, we showed the ability of the resulting MIMO coded modulation schemes to approach the ergodic channel capacity under the practical constraint of finite length codewords. We also showed by simulations that the optimized coded modulation signaling methods approach the outage capacity of quasi-static channels except for the case of $2 \times 1$, BICM with spatial multiplexing, rate $1 / 2$ and 4-QAM. Performance evaluation was not limited to computer simulations with synthetically generated channels. We used a MIMO hardware demonstrator developed at the University of A Coruña to evaluate the MIMO coded modulation methods in realistic environments. Different experiments were carried out at the $5 \mathrm{GHz}$ Industrial, Scientific and Medical (ISM) band 
considering different $\mathrm{Tx} / \mathrm{Rx}$ locations and antenna positions. The results were presented in terms of Block Error Rate (BLER) versus $E_{b} / N_{0}$ at reception and were representative of the performance obtained over a typical indoor scenario.

Both computer simulations and experimental measurements showed that concatenation with STBCs is not necessary in order to retain maximum system capacity and spatial diversity. Simply spreading the output symbols of a BICM system among the different transmit antennas provides the same optimum performance. The utilization of STBCs is only justified to simplify the detection procedure when a large number of antennas and/or a higher modulation format is employed. This is not the case in the scenarios considered in this chapter where the detection complexity of BICM schemes is comparable to those using STBCs.

\section{Acknowledgements}

This work has been supported by Xunta de Galicia through grant 09TIC008105PR and by Ministerio de Ciencia e Innovación of Spain and FEDER funds of the EU under grants TEC2007-68020-C04-01 (MultiMIMO project) and CSD2008-00010 (COMONSENS project).

\section{References}

Alamouti, S. M. (1998). A simple transmit diversity technique for wireless communications, 16: 1451-1458.

Belfiore, J.-C., Reyaka, G. \& Viterbo, E. (2005). The Golden code: A $2 \times 2$ full-rate space-time code with nonvanishing determinants, 51(4): 1432-1436.

Berrou, C., Glavieux, A. \& Thitimajshima, P. (1993). Near Shannon limit error-correcting coding and decoding: Turbo-codes, ICC '93, Geneva, Switzerland, pp. 1064-1070.

Fernández-Caramés, T. M., García-Naya, J. A., González-Lopez, M. \& Castedo, L. (2008). MIMO testbed middleware for transmission automation, Proc. 50th International Symposium ELMAR, Vol. 1, Zadar, Croatia, pp. 215-218.

Forney, Jr., G. D. \& Wei, L. F. (1989). Multidimensional constellations - Part I: Introduction, figures of merit, and generalized cross constellations, 7(6): 877-892.

Gallager, R. G. (1963). Low Density Parity Check Codes, Research Monograph 21. MIT Press, Cambridge, Mass.

Garcia-Frias, J. \& Zhong, W. (2003). Approaching Shannon performance by iterative decoding of linear codes with low-density generator matrix, 7(6): 266-268.

García-Naya, J. A., González-López, M. \& Castedo, L. (2010). Radio Communications, In-Tech, chapter A Distributed Multilayer Software Architecture for MIMO Testbeds.

García-Naya, J. A., Perez-Iglesias, H. J., Fernández-Caramés, T. M., González-López, M. \& Castedo, L. (2008). A distributed multilayer architecture enabling end-user access to MIMO testbeds, Proc. IEEE 19th International Symposium on Personal, Indoor and Mobile Radio Communications, Cannes, France, pp. 1-5.

González-López, M., Vázquez-Araújo, F. J., Castedo, L. \& Garcia-Frias, J. (2006a). Design of Serially-Concatenated Low-Density Generator Matrix codes using EXIT charts, Intl. Symp. on Turbo Codes E Related Topics, Munich, Ger.

González-López, M., Vázquez-Araújo, F. J., Castedo, L. \& Garcia-Frias, J. (2006b). Optimized Serially-Concatenated LDGM and Alamouti codes for approaching MIMO capacity, PIMRC'06, Helsinki, Finland. 
González-López, M., Vázquez-Araújo, F. J., Castedo, L. \& Garcia-Frias, J. (2007). Serially-Concatenated Low-Density Generator Matrix (SCLDGM) codes for transmission over AWGN and Rayleigh fading channels, 6(8): 2753-2758.

Hassibi, B. \& Hochwald, B. M. (2002). High-rate codes that are linear in space and time, 48(7): 1804-1824.

Hochwald, B. M. \& ten Brink, S. (2003). Achieving near-capacity on a multiple-antenna channel, 51(3): 389-399.

IEEE Standard for Local and Metropolitan Area Networks. Part 16: Air Interface for Fixed and Mobile Broadband Wireless Access Systems (2009).

IEEE Standard for Wireless LAN Medium Access Control (MAC) and PHYsical Layer (PHY) Specifications: Amendment: Medium Access Control (MAC) Enhancements for Higher Throughput (2009).

Jin, H., Khandekar, A. \& McEliece, R. J. (2000). Irregular repeat-accumulate codes, Proc. 2nd Int. Symp. Turbo Codes \& Related Topics, Brest, France, pp. 1-8.

Lyrtech (2010).

URL: http://www.lyrtech.com

MacKay, D. J. C. (1999). Good error-correcting codes based on very sparse matrices, 45(2): 399-431.

Sandhu, S. \& Paulraj, A. (2000). Space-time block codes: A capacity perspective, 4(12): 384-386.

Schreckenbach, F., Gortz, N., Hagenauer, J. \& Bauch, G. (2003). Optimized symbol mappings for bit-interleaved coded modulation with iterative decoding, Proc. Global Telecommunications Conference (GLOBECOM 2003), San Francisco, CA, USA.

Sundance Multiprocessor (2010). URL: http://www.sundance.com

Telatar, İ. E. (1995). Capacity of multi-antenna Gaussian channels, Technical report, Bell Laboratories, Lucent Technologies. Published in European Transactions on Telecommunications, Vol. 10, No. 6, pp. 585-595, Nov/Dec 1999.

ten Brink, S. (1999). Convergence of iterative decoding, IEE Electronics Letters 35(13): 1117-1118.

ten Brink, S. (2001). Convergence behavior of iteratively decoded parallel concatenated codes, 49(10): 1727-1737.

ten Brink, S. \& Kramer, G. (2003). Design of Repeat-Accumulate codes for iterative detection and decoding, 51(11): 2764-2772.

ten Brink, S., Kramer, G. \& Ashikhmin, A. (2004). Design of Low-Density Parity-Check codes for modulation and detection, 52(4): 670-678.

Tonello, A. M. (2000). Space-Time Bit-Interleaved Coded Modulation with an iterative decoding strategy, Proc. IEEE VTC Fall 2000, Boston.

Vázquez-Araújo, F. J., González-López, M., Castedo, L. \& Garcia-Frias, J. (2006). Design of Serially-Concatenated LDGM coded MIMO systems, Proc. SPAWC'06, Cannes, France.

Vázquez-Araújo, F. J., González-López, M., Castedo, L. \& Garcia-Frias, J. (2007). Serially-Concatenated LDGM codes for MIMO channels, 6(8): 2860-2871.

Vikalo, H., Hassibi, B. \& Kailath, T. (2004). Iterative decoding for MIMO channels via modified sphere decoding, 3(6): 2299-2311.

Wang, X. \& Poor, H. V. (1999). Iterative (turbo) soft interference cancellation and decoding for coded CDMA, 47(7): 1046-1061. 
Yue, G. \& Wang, X. (2005). Optimization of Irregular Repeat Accumulate codes for MIMO systems with iterative receivers, 4(6): 2843-2855.

Yue, G., Wang, X. \& Madihian, M. (2008). Outage performance and IRA code design for MIMO block fading with unitary scrambling, 54(4): 2354-2363.

Zehavi, E. (1992). 8-PSK trellis codes for a Rayleigh channel, 40: 873-884. 


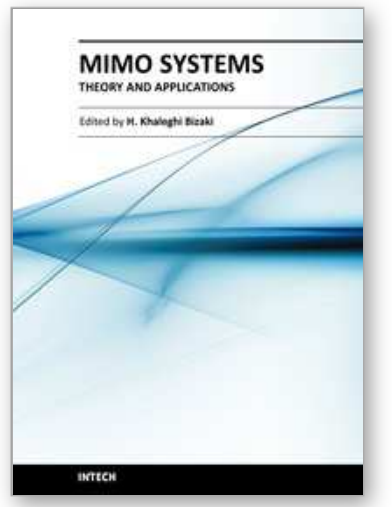

\author{
MIMO Systems, Theory and Applications \\ Edited by Dr. Hossein Khaleghi Bizaki
}

ISBN 978-953-307-245-6

Hard cover, 488 pages

Publisher InTech

Published online 04, April, 2011

Published in print edition April, 2011

In recent years, it was realized that the MIMO communication systems seems to be inevitable in accelerated evolution of high data rates applications due to their potential to dramatically increase the spectral efficiency and simultaneously sending individual information to the corresponding users in wireless systems. This book, intends to provide highlights of the current research topics in the field of MIMO system, to offer a snapshot of the recent advances and major issues faced today by the researchers in the MIMO related areas. The book is written by specialists working in universities and research centers all over the world to cover the fundamental principles and main advanced topics on high data rates wireless communications systems over MIMO channels. Moreover, the book has the advantage of providing a collection of applications that are completely independent and self-contained; thus, the interested reader can choose any chapter and skip to another without losing continuity.

\title{
How to reference
}

In order to correctly reference this scholarly work, feel free to copy and paste the following:

Francisco J. Vázquez Araújo, José A. Garcia-Naya, Miguel González-López, Luis Castedo and Javier GarciaFrias (2011). Experimental Evaluation of MIMO Coded Modulation Systems: are Space-Time Block Codes Really Necessary?, MIMO Systems, Theory and Applications, Dr. Hossein Khaleghi Bizaki (Ed.), ISBN: 978953-307-245-6, InTech, Available from: http://www.intechopen.com/books/mimo-systems-theory-andapplications/experimental-evaluation-of-mimo-coded-modulation-systems-are-space-time-block-codes-reallynecessary

\section{INTECH}

open science | open minds

\section{InTech Europe}

University Campus STeP Ri

Slavka Krautzeka 83/A

51000 Rijeka, Croatia

Phone: +385 (51) 770447

Fax: +385 (51) 686166

www.intechopen.com

\section{InTech China}

Unit 405, Office Block, Hotel Equatorial Shanghai

No.65, Yan An Road (West), Shanghai, 200040, China 中国上海市延安西路65号上海国际贵都大饭店办公楼 405 单元

Phone: +86-21-62489820

Fax: +86-21-62489821 
(C) 2011 The Author(s). Licensee IntechOpen. This chapter is distributed under the terms of the Creative Commons Attribution-NonCommercialShareAlike-3.0 License, which permits use, distribution and reproduction for non-commercial purposes, provided the original is properly cited and derivative works building on this content are distributed under the same license. 